\title{
TRAF2 is a biologically important necroptosis suppressor
}

\author{
SL Petersen ${ }^{1}$, TT Chen ${ }^{1}$, DA Lawrence ${ }^{1}$, SA Marsters ${ }^{1}$, F Gonzalvez $^{1}$ and A Ashkenazi ${ }^{\star * 1}$
}

Tumor necrosis factor $\alpha$ (TNF $\alpha$ ) triggers necroptotic cell death through an intracellular signaling complex containing receptorinteracting protein kinase (RIPK) 1 and RIPK3, called the necrosome. RIPK1 phosphorylates RIPK3, which phosphorylates the pseudokinase mixed lineage kinase-domain-like (MLKL)—driving its oligomerization and membrane-disrupting necroptotic activity. Here, we show that TNF receptor-associated factor 2 (TRAF2)-previously implicated in apoptosis suppression-also inhibits necroptotic signaling by TNF $\alpha$. TRAF2 disruption in mouse fibroblasts augmented TNF $\alpha$-driven necrosome formation and RIPK3-MLKL association, promoting necroptosis. TRAF2 constitutively associated with MLKL, whereas TNF $\alpha$ reversed this via cylindromatosis-dependent TRAF2 deubiquitination. Ectopic interaction of TRAF2 and MLKL required the C-terminal portion but not the N-terminal, RING, or CIM region of TRAF2. Induced TRAF2 knockout (KO) in adult mice caused rapid lethality, in conjunction with increased hepatic necrosome assembly. By contrast, TRAF2 KO on a RIPK3 KO background caused delayed mortality, in concert with elevated intestinal caspase-8 protein and activity. Combined injection of TNFR1-Fc, Fas-Fc and DR5-Fc decoys prevented death upon TRAF2 KO. However, Fas-Fc and DR5-Fc were ineffective, whereas TNFR1-Fc and interferon $\alpha$ receptor (IFNAR1)-Fc were partially protective against lethality upon combined TRAF2 and RIPK3 KO. These results identify TRAF2 as an important biological suppressor of necroptosis in vitro and in vivo.

Cell Death and Differentiation (2015) 22, 1846-1857; doi:10.1038/cdd.2015.35; published online 17 April 2015

Apoptotic cell death is mediated by caspases and has distinct morphological features, including membrane blebbing, cell shrinkage and nuclear fragmentation. ${ }^{1-4}$ In contrast, necroptotic cell death is caspase-independent and is characterized by loss of membrane integrity, cell swelling and implosion. ${ }^{1,2,5}$ Nevertheless, necroptosis is a highly regulated process, requiring activation of RIPK1 and RIPK3, which form the core necrosome complex. ${ }^{1,2,5}$ Necrosome assembly can be induced via specific death receptors or toll-like receptors, among other modules. ${ }^{6-9}$ The activated necrosome engages MLKL by RIPK3-mediated phosphorylation. ${ }^{6,10,11}$ MLKL then oligomerizes and binds to membrane phospholipids, forming pores that cause necroptotic cell death. ${ }^{10,12-15}$ Unchecked necroptosis disrupts embryonic development in mice and contributes to several human diseases. ${ }^{7,8,16-22}$

The apoptotic mediators FADD, caspase- 8 and cFLIP suppress necroptosis. ${ }^{19-21,23,24}$ Elimination of any of these genes in mice causes embryonic lethality, subverted by additional deletion of RIPK3 or MLKL. ${ }^{19-21,25}$ Necroptosis is also regulated at the level of RIPK1. Whereas TNFa engagement of TNFR1 leads to K63-linked ubiquitination of RIPK1 by cellular inhibitor of apoptosis proteins (cIAPs) to promote nuclear factor (NF)- $\kappa \mathrm{B}$ activation, ${ }^{26}$ necroptosis requires suppression or reversal of this modification to allow RIPK1 autophosphorylation and consequent RIPK3 activation. ${ }^{2,23,27,28}$ CYLD promotes necroptotic signaling by deubiquitinating RIPK1, augmenting its interaction with RIPK3. ${ }^{29}$ Conversely, caspase-8-mediated CYLD cleavage inhibits necroptosis. ${ }^{24}$

TRAF2 recruits clAPs to the TNFa-TNFR1 signaling complex, facilitating NF- $k$ B activation. ${ }^{30-33}$ TRAF2 also supports K48-linked ubiquitination and proteasomal degradation of death-receptor-activated caspase-8, curbing apoptosis. ${ }^{34}$ TRAF2 KO mice display embryonic lethality; some survive through birth but have severe developmental and immune deficiencies and die prematurely. ${ }^{35,36}$ Conditional TRAF2 KO leads to rapid intestinal inflammation and mortality. ${ }^{37}$ Furthermore, hepatic TRAF2 depletion augments apoptosis activation via Fas/CD95. ${ }^{34}$ TRAF2 attenuates necroptosis induction in vitro by the death ligands Apo2L/TRAIL and Fas/CD95L. ${ }^{38}$ However, it remains unclear whether TRAF2 regulates TNFainduced necroptosis-and if so-how. Our present findings reveal that TRAF2 inhibits TNFa necroptotic signaling. Furthermore, our results establish TRAF2 as a biologically important necroptosis suppressor in vitro and in vivo and provide initial insight into the mechanisms underlying this function.

\section{Results}

Loss of TRAF2 promotes TNFa-induced necroptosis. To investigate whether TRAF2 regulates necroptosis, we first

${ }^{1}$ Cancer Immunology, Genentech, Inc., 1 DNA Way, South San Francisco, CA 94080, USA

*Corresponding author: A Ashkenazi, Cancer immunology, Genentech, Inc., 1 DNA Way, MS 42, South San Francisco, CA 94080-4918, USA. Tel: +1 650 225 1853; Fax: +1 6504678195 ; E-mail: aa@gene.com

Abbreviations: $\mathrm{CHX}(\mathrm{C})$, cycloheximide; CIAP, cellular inhibitor of apoptosis protein; CYLD, cylindromatosis; FADD, Fas-associated death domain; IFN, interferon; MEF, mouse-embryo fibroblast; MLKL, mixed lineage kinase domain-like; Nec-1 (N), necrostatin-1; NF- $\kappa B$, nuclear factor- $-\mathrm{B}$; RIPK1, receptor interacting protein kinase 1; RIPK3, receptor interacting protein kinase 3; TNF $\alpha(\mathrm{T})$, tumor necrosis factor $\alpha$; TRAF2, TNF receptor-associated factor 2; Ub, ubiquitin; Z-VAD-fmk (Z), benzyloxycarbonyl-Val-Ala-Asp(OMe)-fluoromethylketone

Received 11.11.14; revised 11.2.15; accepted 24.2.15; Edited by G Melino; published online 17.4.15 


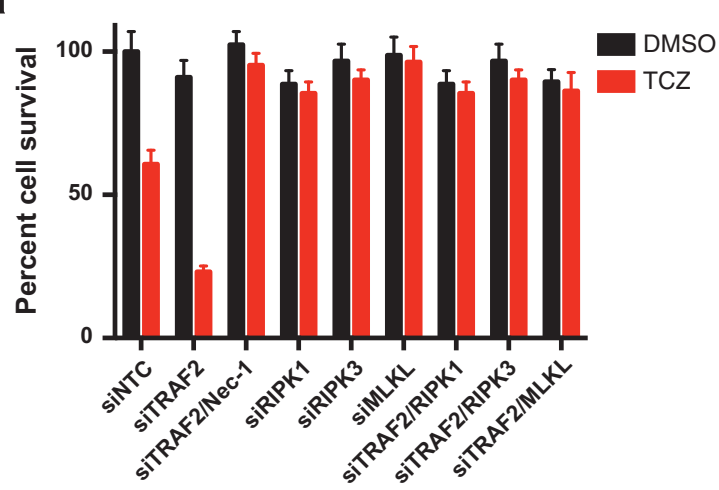

C

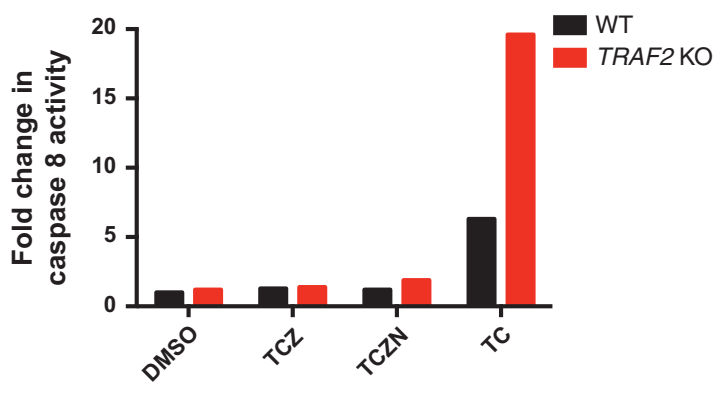

e

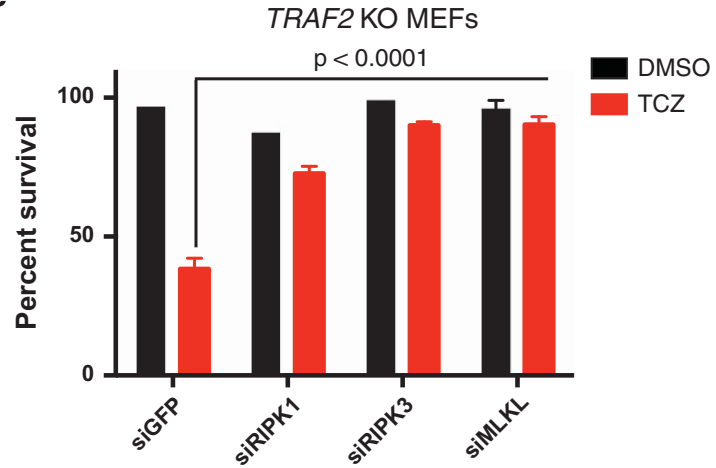

b

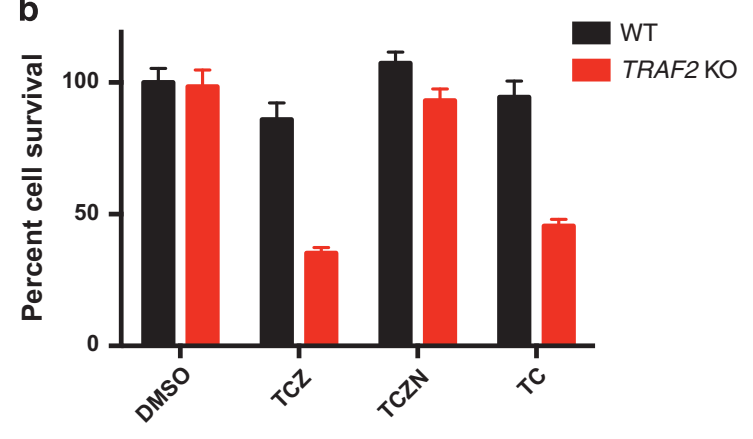

d

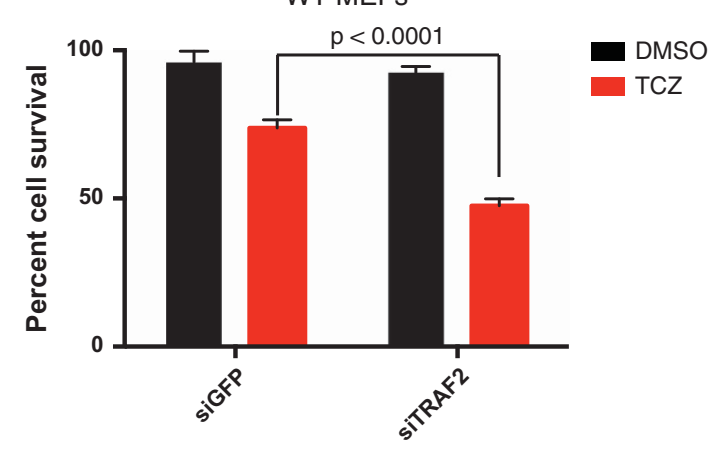

f
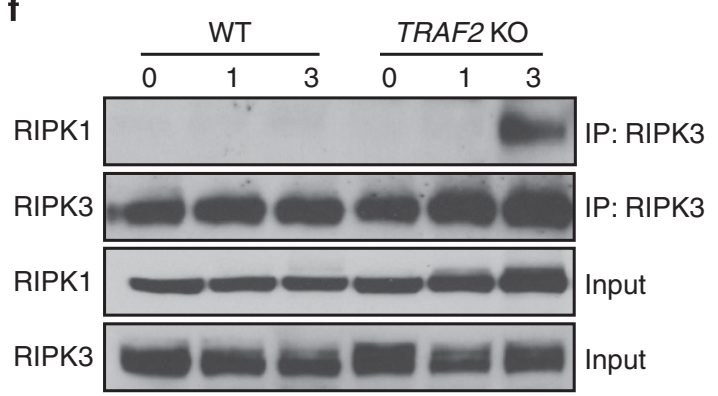

Figure 1 Loss of TRAF2 augments TNF $\alpha$-induced necroptosis. (a) L929 cells were transfected for 3 days with siRNA as indicated. Cells were then pretreated for $1 \mathrm{~h}$ with zVAD (Z) $(20 \mu \mathrm{M})$, cycloheximide (c) $(1 \mu \mathrm{g} / \mathrm{ml})$ and necrostatin-1 (N) $(20 \mu \mathrm{M})$ for $1 \mathrm{~h}$ followed by TNF $\alpha$ (T) $(100 \mathrm{ng} / \mathrm{ml})$ for an additional $5 \mathrm{~h}$. Cell survival was determined by measuring ATP consumption using Cell Titer Glo assay. (b, c) WT and TRAF2 KO MEFs were treated and analyzed for viability (b) or for caspase-8 activity (c). (d, e) WTand TRAF2 KO MEFs were transfected with siRNA for 3 days, subjected to the indicated treatments and analyzed for survival. (f) WTand TRAF2 KO MEFs were treated with TCZ as above for the indicated times and analyzed by immunoprecipitation (IP) followed by immunoblot (IB) for proteins indicated on the left. Data in panels a-e represent mean \pm S.E.M. $(n=4)$. $P$ values are by Student's $t$ test

depleted it by siRNA transfection in mouse L929 fibrosarcoma cells. ${ }^{39}$ TRAF2 knockdown significantly augmented TNFa-induced necroptosis, evident by loss of cell viability in the presence of TNFa plus the translation inhibitor $\mathrm{CHX}$ and the pan-caspase inhibitor Z-VAD-fmk (TCZ); and by inhibition of cell death by the RIPK1 inhibitor Nec-1 (Figure 1a). Additional siRNA knockdown of RIPK1, RIPK3 or MLKL reversed the augmentation (Figure 1a and Supplementary Figures $1 \mathrm{~A}$ and $\mathrm{B}$ ), confirming necroptosis suppression.

To rule out potential off-target TRAF2 siRNA effects, we turned next to TRAF2 KO MEFs. TRAF2 KO MEFs were much more responsive to TCZ-induced death than were wild-type (WT) MEFs (Figure 1b). Nec-1 blocked cell death in both cases, verifying necroptosis augmentation upon TRAF2 deletion. In the absence of Z-VAD (TC), TRAF2 KO MEFs showed stronger TNFa-induced cell death and casapse-8 activation than did WT MEFs (Figures $1 \mathrm{~b}$ and $\mathrm{c}$ ), consistent with earlier evidence that TRAF2 suppresses cell-extrinsic apoptotic signaling. ${ }^{34,40}$ TRAF2 siRNA knockdown in WT MEFs also enhanced TNFa-induced necroptosis (Figure 1d and Supplementary Figure 1C). Knockdown of RIPK1, RIPK3 or MLKL attenuated TCZ-induced death in TRAF2 KO MEFs, suggesting that TRAF2 regulates necroptosis via the 
RIPK1-RIPK3-MLKL pathway (Figure 1e and Supplementary Figures $1 \mathrm{D}$ and $\mathrm{E})$. TRAF2 KO MEFs formed a readily detectable necrosome, evident by co-immunoprecipitation (co-IP) of RIPK3 within $3 \mathrm{~h}$ of TCZ treatment (Figure $1 \mathrm{f}$ and
Supplementary Figures 1F and G); WT MEFs assembled a weaker necrosome by $6 \mathrm{~h}$ (Supplementary Figure $1 \mathrm{H}$ ). Knockdown of TRAF2 in L929 cells similarly augmented RIPK1-RIPK3 association (Supplementary Figure 1I). Thus,

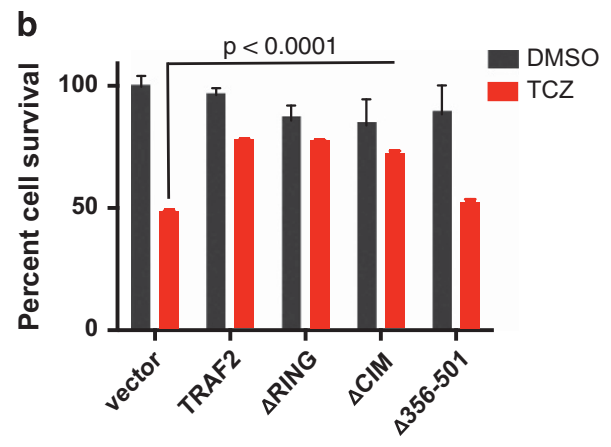

d

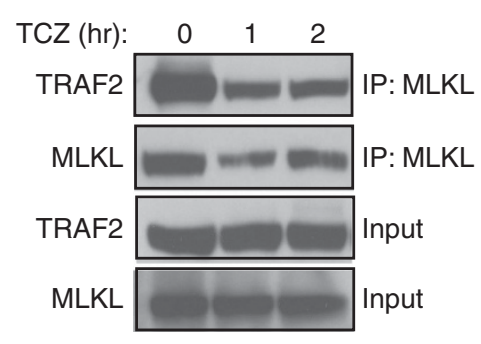

g
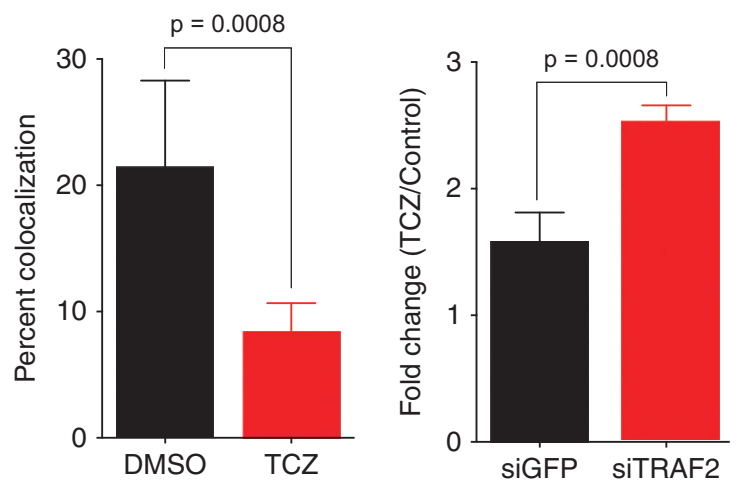
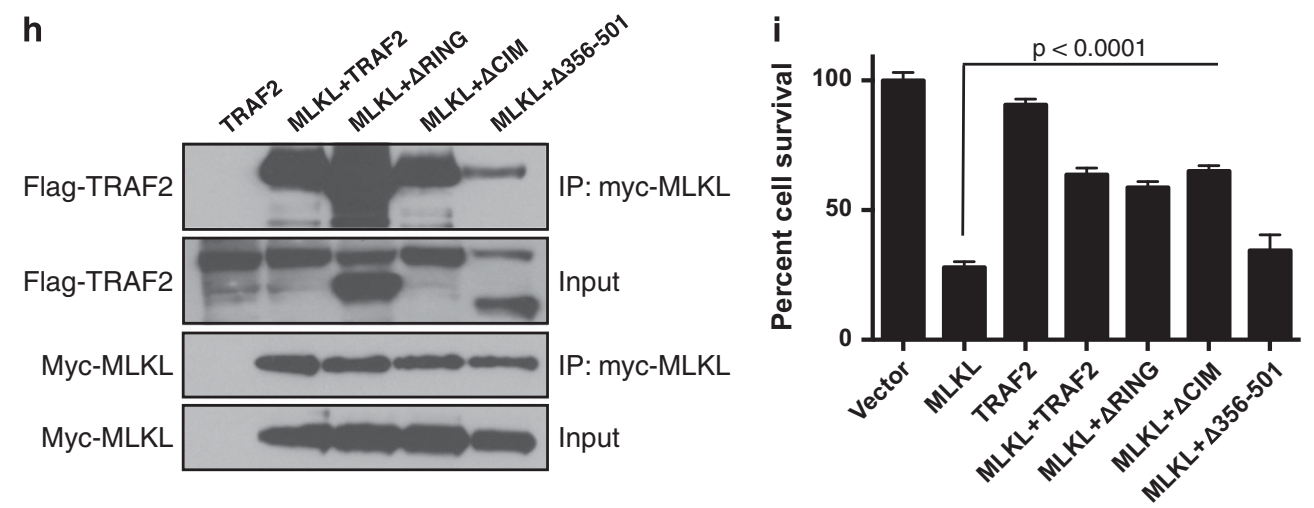
TRAF2 depletion in murine fibroblasts augments TCZ-induced necrosome assembly and necroptosis, suggesting that at least one level of signal suppression by TRAF2 is upstream of the necrosome.

Evidence for a constitutive interaction of TRAF2 with MLKL. Although in the absence of CHX TNFa did not induce detectable cell death in WT MEFs at $5 \mathrm{~h}$ (data not shown), it decreased cell viability by $\sim 50 \%$ within $24 \mathrm{~h}$ (Figure 2a). Both TRAF2 KO and CIAP1 KO MEFS showed greater sensitivity to TNFa than did WT MEFs; however, whereas Z-VAD fully blocked TNFa-induced death in TRAF2 KO MEFs, it provided little protection in clAP1 KO MEFs. Conversely, Nec-1 inhibited TNFa-induced death more strongly in clAP1 $\mathrm{KO}$ as compared with TRAF2 KO MEFs. Thus, in response to TNFa without $\mathrm{CHX}$, TRAF2 $\mathrm{KO}$ favors apoptosis, whereas CIAP1 $\mathrm{KO}$ supports necroptosis, suggesting that that TRAF2 modulates necroptosis not only via clAPs but also at some additional level(s).

Transfection of TRAF2 KO MEFs with a TRAF2 deletion mutant lacking the RING domain suppressed TNFa-induced necroptosis as effectively as did reconstitution with WT TRAF2 (Figure $2 \mathrm{~b}$ and Supplementary Figure 2A). Hence, unlike apoptosis inhibition, ${ }^{34}$ necroptosis suppression by TRAF2 is RING-independent. A TRAF2 mutant lacking the clAPinteraction motif $(\mathrm{CIM})^{30,41}$ was similarly effective at attenuating the effect of TRAF2 $\mathrm{KO}$, consistent with the notion that TRAF2 also operates independently of clAP binding. In contrast, deletion of the C-terminal portion of TRAF2 (aa 356501) did not significantly rescue the TRAF2 KO phenotype.

In light of previous work suggesting that TRAF2 is capable of binding MLK3, ${ }^{42}$ we examined whether TRAF2 also interacts with MLKL. IP of MLKL from WT MEFs revealed association with TRAF2 at baseline, whereas TCZ treatment decreased this interaction by $\sim 50 \%$ over $2 \mathrm{~h}$ (Figure $2 \mathrm{c}$ and Supplementary Figures 2B-D). We detected minimal baseline RIPK3-MLKL interaction in WT MEFs; however, in TRAF2 KO MEFs, RIPK3 and MLKL showed some interaction without stimulation-and this increased significantly upon TCZ treatment, in concert with apparent dissociation of TRAF2 from MLKL (Figure 2c). TRAF2 similarly associated with MLKL in L929 cells, whereas TCZ treatment substantially decreased this interaction (Figure 2d). Consistent with earlier data, ${ }^{10}$ we could not detect RIPK3-MLKL binding in L929 cells.

In keeping with the co-IP data (Figure 2d), immunofluorescence analysis of L929 cells revealed $\sim 20 \%$ co-localization of TRAF2 and MLKL at baseline, whereas TCZ treatment decreased this by $\sim 2.5$-fold (Figure $2 \mathrm{e}$ and Supplementary Figure 2E). Furthermore, siRNA knockdown of TRAF2 increased the co-localization between RIPK3 and MLKL by $\sim 2.5$-fold (Figures $2 f$ and g). Thus, TRAF2 may associate with MLKL at baseline, whereas TNFa stimulation may reverse this in concert with increased RIPK3-MLKL binding, suggesting that TRAF2 association suppresses the interaction of MLKL with RIPK3.

To examine which part of the TRAF2 protein is required for MLKL interaction, we transfected 293T cells with vectors encoding myc-epitope-tagged MLKL together with WT or deletion variants of TRAF2. IP of MLKL via myc revealed association with WT TRAF2 or its RING- or CIM-deletion variants, but not the TRAF2 C-terminal-deletion mutant (Figure 2h). Transfection of MLKL, but not of TRAF2, induced marked cell death (Figure 2i); importantly, co-transfection of MLKL with WT TRAF2 or its RING- or CIM-deletion variants (which retained MLKL binding) substantially blocked celldeath induction, whereas co-transfection with the C-terminaldeletion mutant (which failed to bind MLKL) did not. Cells co-transfected with MLKL and TRAF2 expressed less TRAF2 than did cells transfected with TRAF2 alone, perhaps owing to decreased viability upon MLKL overexpression. Regardless, the C-terminal portion of TRAF2 appears specifically important for MLKL association and for effective necroptosis suppression.

CYLD is required for TRAF2 deubiquitination and dissociation from MLKL. We examined whether the ubiquitination state of TRAF2 affects its apparent interaction with MLKL. IP of TRAF2 from L929 cells under denaturing conditions - to eliminate associated proteins-followed by immunoblot (IB) for ubiquitin, revealed abundant TRAF2 ubiquitination at baseline; however, TCZ treatment completely reversed this within $2 \mathrm{~h}$ (Figure $3 \mathrm{a}$ ). Albeit less robustly, IP of TRAF2 from WT MEFs also indicated basal TRAF2 ubiquitination, which decreased upon TCZ treatment (Supplementary Figure 3A); in contrast, TRAF2 IP from TRAF2 KO MEFs did not show any ubiquitination. IP of RIPK1 indicated RIPK1 association with TRAF2 within $1 \mathrm{~h}$ of TCZ treatment, followed by dissociation by $2 \mathrm{~h}$ (Supplementary Figure 3B), consistent with TNFR1 recruitment and release of RIPK1. As expected, RIPK1 IP revealed its transient ubiquitination, appearing $1 \mathrm{~h}$ after TCZ addition and declining by $2 \mathrm{~h}$ (Supplementary Figure 3C).

CYLD promotes TNFa-induced necroptosis by deubiquinating RIPK $1,{ }^{29}$ yet inhibits CD40 signaling to NF- $\kappa$ B by deubiquinating TRAF2.43,44 To determine whether CYLD regulates TRAF2 in the context of TNFa-induced necroptosis, we depleted CYLD by siRNA in L929 cells. Whereas TCZ treatment induced TRAF2 deubiquitination, CYLD knockdown

\footnotetext{
Figure 2 TRAF2 constitutively interacts with MLKL to suppress necroptosis. (a) WT, TRAF2 KO and cIAP1 KO MEFs were treated as indicated for 1 day and analyzed for survival. (b) TRAF2 KO MEFs were transfected with the indicated expression constructs for 2 days, pretreated with $\mathrm{CZ}$ for $1 \mathrm{~h}$ followed by T for an additional $5 \mathrm{~h}$, and analyzed for survival. (c) WT and TRAF2 KO MEFs were treated as in $\mathbf{b}$ for the indicated times and analyzed by IP and IB. (d) L929 cells were treated as in $\mathbf{c}$ and analyzed by IP and IB. (e) L929 cells were treated as indicated for 90 min and analyzed by immunofluorescence (IF) with specific antibodies to TRAF2 (green) or MLKL (red). (f) Quantitation of IF data generated as in e. (g) L929 cells were transfected for 3 days with siRNA as indicated, treated with DMSO or TCZ for 90 min, analyzed by IF as in e and the data was quantitated $(n=3)$ and depicted as fold change from DMSO control levels. (h) 293T cells were transfected with constructs encoding myc-epitope-tagged MLKL, WT TRAF2 or indicated TRAF2 deletion mutants for 1 day followed by myc IP and TRAF2 IB. (i) 293T cells were transfected as in $\mathbf{h}$ for 2 days and analyzed for survival. Bar graphs in i depict mean \pm S.E. M. of triplicates. Data in panels $\mathbf{a}, \mathbf{b}$ depict mean \pm S.E.M. $(n=4)$. For colocalization studies (panels $\mathbf{f}$ and $\mathbf{g}$ ) three independent experiments were performed and six images for each treatment were quantified. $P$ values are by Student's $t$ test
} 
reversed this effect and stabilized the association of TRAF2 with MLKL (Figures 3b and c). In keeping with earlier data, ${ }^{29}$ CYLD knockdown provided complete protection against TNFa-induced necroptosis (Figure 3d). Importantly, CYLD depletion also blocked cell-death augmentation by TRAF2 knockdown (Figure 3d). Furthermore, whereas clAP1mediated in vitro ubiquitination of recombinant $(r)$ TRAF2 supported binding to $\mathrm{rMLKL}$, addition of $\mathrm{rCYLD}$ reversed rTRAF2 ubiquitination and partially inhibited rTRAF2 binding to rMLKL (Supplementary Figure 3D). Thus, TRAF2 deubiquitination by CYLD promotes the dissociation of TRAF2 from MLKL.

TRAF2 $\mathrm{KO}$ is lethal in adult mice. Mouse embryonic lethality of TRAF2 KO has been ascribed to deficient NF- $k \mathrm{~B}$ signaling and excessive apoptosis. ${ }^{35,36}$ The observation that loss of TRAF2 augments necroptosis in vitro prompted us to investigate whether elevated necroptosis also contributes to in vivo mortality upon deletion of TRAF2. To examine this, we generated inducible TRAF2 KO mice via a TRAF2 loxp/loxp. RosaCreERTS gene construct (see Methods). To induce ubiquitous TRAF2 deletion, we treated mature mice with tamoxifen at 40 or $80 \mathrm{mg} / \mathrm{kg}$ daily for 5 days by intraperitoneal injection. All tamoxifen-treated mice displayed severe weight loss, deterioration in vital appearance of the liver and gastrointestinal system, and moribund behavior; these mice died or had to be killed within 1-3 days following final tamoxifen injection (Figures $4 a$ and $c$ ). To rule out potential off-target effects of tamoxifen, we compared cre-negative and cre-positive mice possessing both loxp sites. In response to tamoxifen $(40 \mathrm{mg} / \mathrm{kg})$, cre-negative mice displayed no adverse reactions, whereas cre-positive mice lost significant a

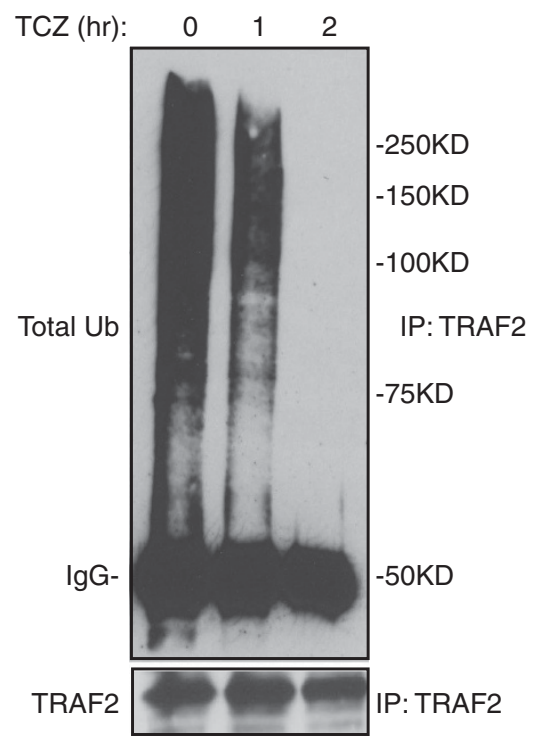

C

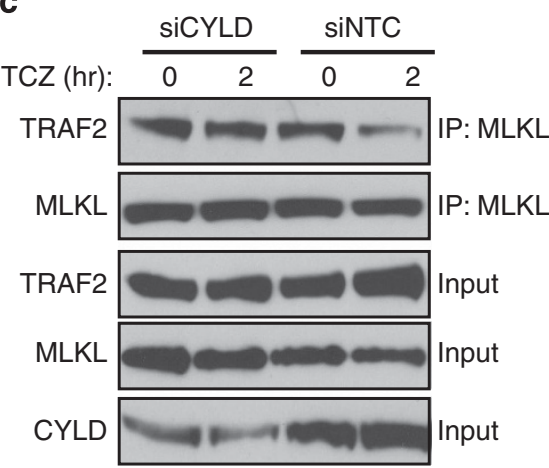

b

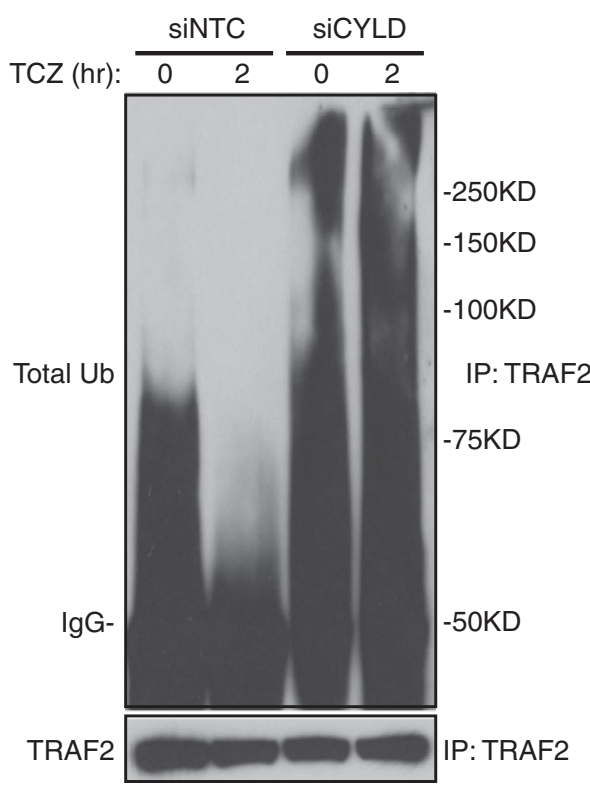

d

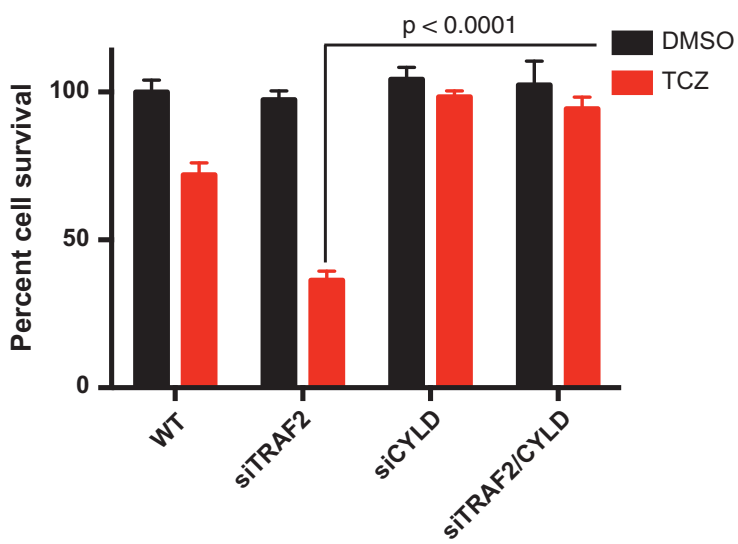

Figure 3 CYLD mediates TRAF2 deubiquitination and dissociation from MLKL. (a) L929 cells were treated for with CZ for $1 \mathrm{~h}$ followed by T for the indicated times. Cells were lysed in $1.5 \%$ SDS, diluted to $0.15 \%$ in SDS-PAGE buffer and analyzed by TRAF2 IP followed by IB with anti-ubiquitin (Ub) antibody. (b) L929 cells were transfected with siRNA as indicated for 3 days followed by treatment and analysis as in a. (c) L929 cells were transfected as in b, pretreated with CZ for $1 \mathrm{~h}$ followed by T for indicated times, and analyzed by IP and IB. (d) L929 cells were transfected as in $\mathbf{b}$, treated with CZ for $1 \mathrm{~h}$ followed with $\mathrm{T}$ for $5 \mathrm{~h}$, and analyzed for survival. Data in $\mathbf{d}$ depict mean \pm S.E.M. ( $n=4$ ). $P$ values are by Student's $t$ test 
a

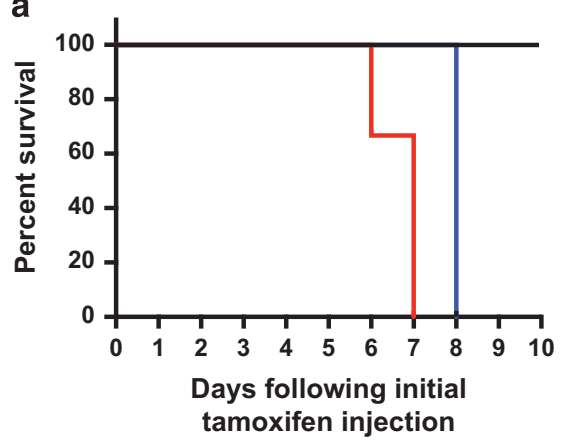

b

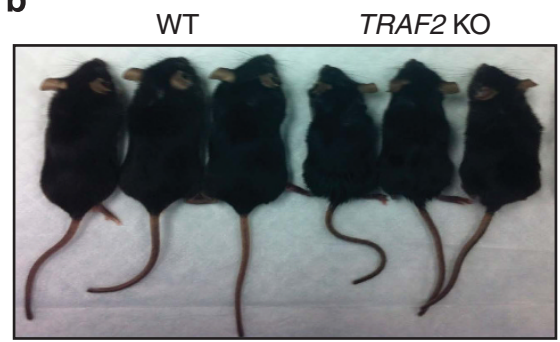

d

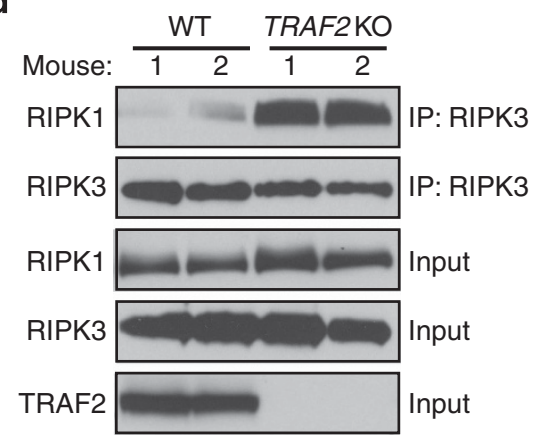

— Vehicle
— Tamoxifen $(40 \mathrm{mg} / \mathrm{kg})$
$\leftarrow$ Tamoxifen $(80 \mathrm{mg} / \mathrm{kg})$

c

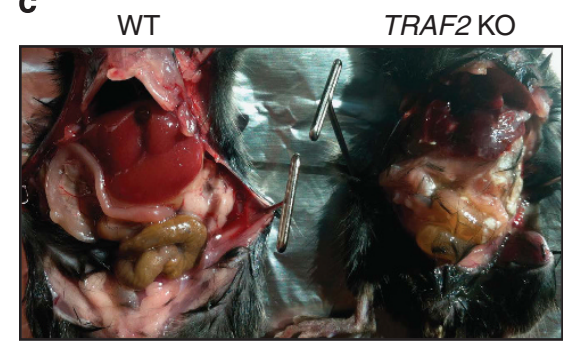

e

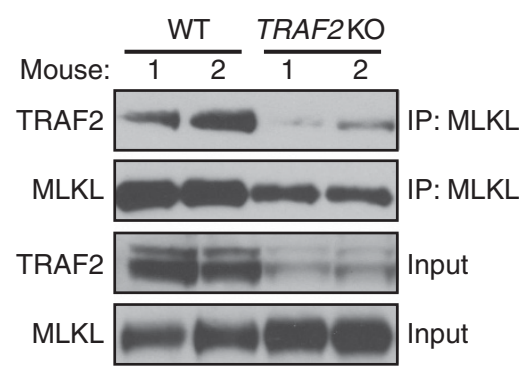

Figure 4 Inducible TRAF2 KO in adult mice causes lethality in conjunction with enhanced hepatic necrosome formation. (a) Inducible TRAF2 KO mice ( $n=5)$ were injected i. p. with vehicle or tamoxifen at the indicated doses daily for 5 days followed by observation for morbidity. (b, c) Representative image of overall external (b) and internal abdominal cavity (c) condition. (d, e) WT and TRAF2 KO mice were injected with tamoxifen as in a. Livers were collected 3 days after the final tamoxifen injection (day 8), extracted and analyzed for necrosome formation (d) and TRAF2-MLKL association (e) by IP and IB as indicated

weight and had to be killed or died within 3 days of final injection (day 8) (Supplementary Figures 4A-C). To determine whether necroptosis contributed to morbidity, we harvested livers from WT and TRAF2 KO mice at day 8 and examined tissue extracts for necrosome formation. Whereas necrosome assembly was not detectable in WT livers, it was readily observable in livers from TRAF2 KO mice (Figure 4d). Importantly, MLKL IP revealed interaction with TRAF2 in livers from WT but not TRAF2 KO mice (Figure 4e). We were unable to measure necrosome formation in intestinal tissue extracts (Supplementary Figure 4D). Thus, TRAF2 deletion in vivo causes morbidity in conjunction with enhanced hepatic necrosome formation, suggesting that excessive necroptosis might contribute to lethality.

RIPK3 KO delays TRAF2 KO mortality. To assess the latter more directly, we generated the same inducible TRAF2 KO on a RIPK3 KO background, by crossing TRAF2 loxp/loxp
RosaCre ERTS mice with RIPK3.neono.ko mice (see Methods). This cross did not result in gross developmental or postnatal abnormality (data not shown). However, upon induction of TRAF2 deletion by tamoxifen $(20 \mathrm{mg} / \mathrm{kg}$ per day daily over 5 days), TRAF2/RIPK3 double-KO (DKO) mice displayed a significant delay in mortality relative to TRAF2 KO mice, with a median survival of 99 versus $30 \mathrm{~h}$ following final tamoxifen dosing (Figures $5 \mathrm{a}-\mathrm{c}$ and Supplementary Figure 4E). DKO mice did ultimately die, displaying deterioration in vital appearance of the liver and gastrointestinal system. Surprisingly, whereas intestinal tissue extracts from WT and TRAF2 KO mice did not have detectable procaspase8 (p55/53) protein, extracts from DKO mice showed elevated procaspase-8 as well as cleaved caspase-8 (p43/41) (Figures $5 \mathrm{~d}$ and e). Intestinal extracts from DKO mice also showed an increase in caspase- 8 mRNA levels as compared with those from WT or TRAF2 KO mice (Supplementary Figure 4F). Consistent with this, intestinal extracts from WT 
a

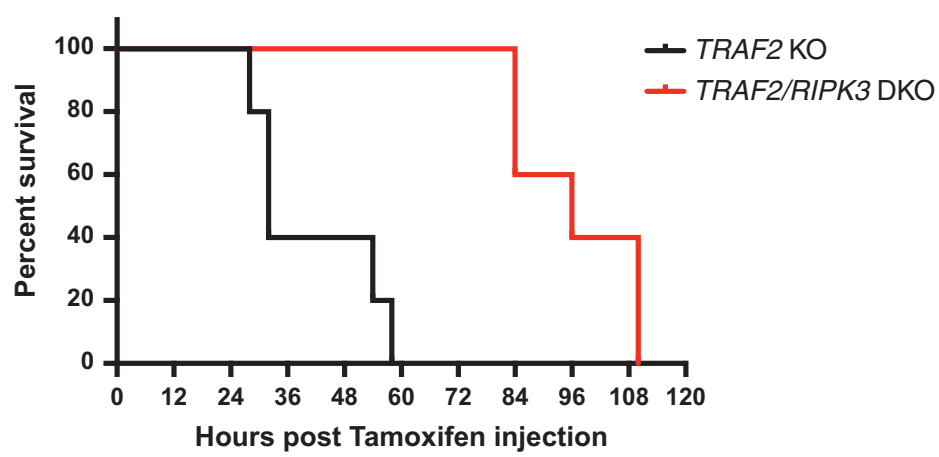

b

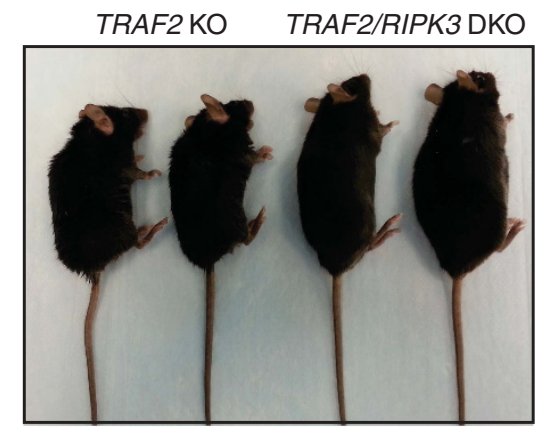

d

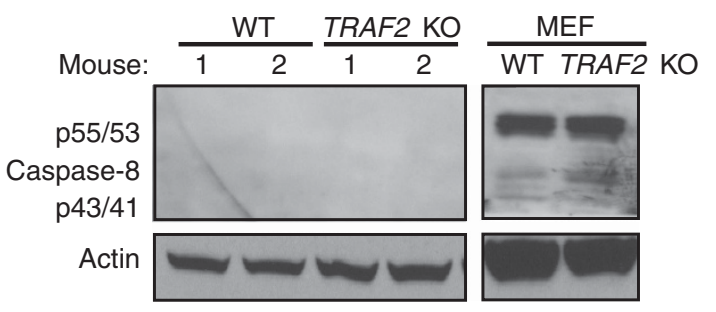

f

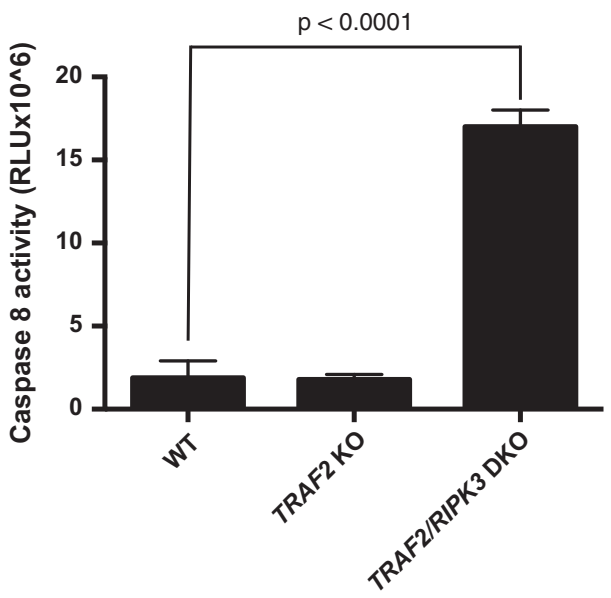

c

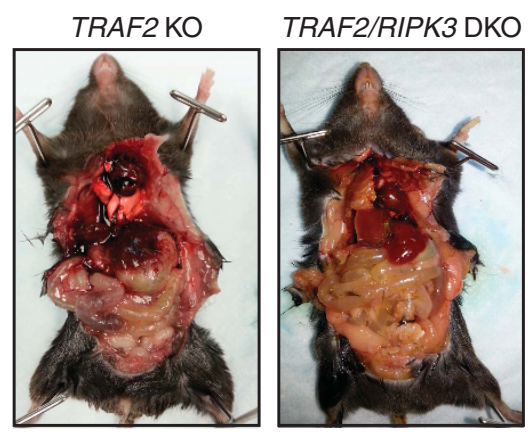

e
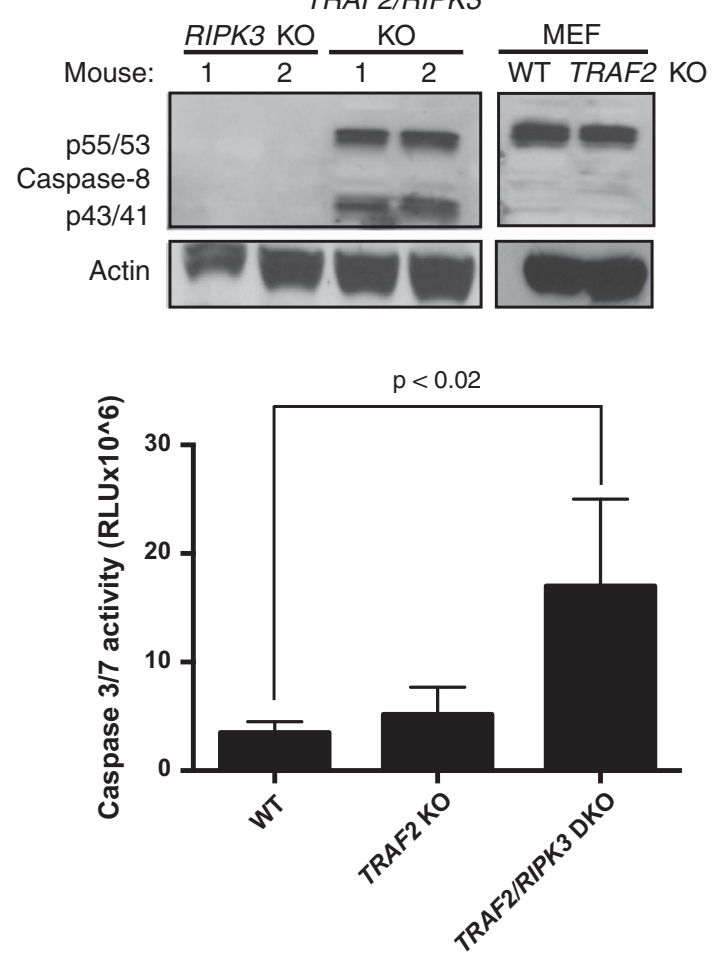

Figure 5 RIPK3 KO delays TRAF2 KO lethality but augments intestinal caspase-8 activity. (a) Mice with inducible TRAF2 KO on a RIPK3 WT or a RIPK3 KO background (DKO) were injected i.p. with tamoxifen (20 mg/kg) daily for 5 days and observed for morbidity $(n=5, P=0.006)$. (b, c) Representative image of overall external condition (b) and internal abdominal cavity (c). (d, e) WT and TRAF2 KO mice (d) or RIPK3KO and TRAF2/RIPK3 DKO mice (e) were injected with tamoxifen as in a. Intestines were collected on day 8 (or day 7 if signs of morbidity appeared) and intestinal extracts were analyzed by caspase-8 IB in comparison with equal protein amounts. (f) WT, TRAF2 KO and TRAF2/ RIPK3 DKO mice were injected as in $\mathbf{a}$. Intestinal extracts were collected as in $\mathbf{d}$ and $\mathbf{e}$ and analyzed by caspase-8 and caspase-3/7 activity assay. Bar graphs depict mean \pm S.E.M. $(n=4)$. $P$ values are by log-rank (Mantel-Cox) test (a) or Student's $t$ test (f) 
and TRAF2 KO mice showed minimal caspase-8 and caspase-3/7 activity, whereas DKO mice displayed higher activity, especially of caspase-8 (Figure 5f). Furthermore, hepatic extracts from either mouse genotype showed little difference in total caspase-8 and caspase-3/7 levels or activity (Supplementary Figures $4 \mathrm{G}$ and $\mathrm{H}$ ). Thus, although necroptosis in the liver (and perhaps other tissues not examined) may drive lethality in TRAF2 KO mice, intestinal apoptosis may be more pathologically dominant in TRAF2/ RIPK3 DKO mice.

Combined neutralization of death ligands differentially impacts lethality. To examine whether additional death receptors besides TNFR1, namely DR5 and Fas, mediate necroptosis in vivo, we used Fc-fusion decoys based on each receptor's ligand-binding ectodomain. We injected each decoy protein or a CD4-Fc control at $10 \mu \mathrm{g}$ per day during the 5 days of tamoxifen treatment. TNFR1-Fc, DR5-Fc and Fas-Fc each delayed TRAF2 KO lethality, extending median survival by 3,2 or 1 day, respectively, relative to CD4-Fc (Figure 6a). Importantly, combined treatment with all three death-receptor Fc fusions fully rescued TRAF2 KO mice from weight loss and lethality for at least 3 weeks (Figure $6 \mathrm{~b}$ and Supplementary Figures $5 \mathrm{~A}$ and $\mathrm{B}$ ). Moreover, combined Fc treatment also blocked necrosome formation in the liver (Supplementary Figure 5C). In marked contrast, combined Fc administration during tamoxifen treatment did not preventand, at best, delayed-mortality in TRAF2/RIPK3 DKO mice (Figure 6c). TNFR1-Fc alone or combined Fc injection similarly increased median survival from 4 to 6 days (Figures $6 \mathrm{c}$ and $\mathrm{d}$ ). Together, these results suggest that upon TRAF2 KO on a WT background, integrated death-ligand signaling is necessary and sufficient to drive mortality. In contrast, upon TRAF2 KO on a RIPK3 KO background, although TNFR1 signaling appears to contribute to lethality early on, other signals-independent of DR5 or Faseventually drive death. Previous work suggests that type I IFN promotes apoptosis independently of death ligands. ${ }^{45}$ Injection of IFNa receptor type 1 (IFNAR1-Fc) together with TNFR1-Fc further extended survival by about 4 days (Figure 6e) and diminished caspase-8 and caspase-3/7 activity in intestinal extracts (Figure 6f). Thus, IFNAR1 significantly contributes along with TNFRI to the lethality of TRAF2 KO on a RIPK3-null background, but additional pathological driver(s) must exist.

\section{Discussion}

Necroptosis appears to act as a fail-safe mechanism for apoptosis. ${ }^{39,46}$ A number of critical mediators of necroptotic signaling downstream of death receptors have been identified, including RIPK1, RIPK3 and MLKL. Several components of the NF- $K B$ and the cell-extrinsic apoptosis pathways, including clAPS, CYLD and caspase-8, exert important regulatory controls on necroptotic signaling via TNFR1.24,29 Previous work implicates TRAF2 as an adaptor that binds to TNFR1 upon ligation by TNFa and recruits clAPs into the membrane-proximal signaling complex to promote NF- $\kappa \mathrm{B}$ activation. ${ }^{31,33,41,47}$ TRAF2 inhibits TNFa-induced apoptosis independent of its role in NF- $\kappa$ B activation. ${ }^{40,48}$ TRAF2 also suppresses death-receptor-mediated apoptosis by promoting K48-ubiquitination and proteasomal degradation of activated caspase-8. ${ }^{34}$ The present findings demonstrate that TRAF2 plays an additional role in curbing necroptotic TNF $a$ signaling by acting both upstream and downstream of the necrosome. Our investigation establishes an important role for TRAF2 as a necroptosis suppressor in vitro and in vivo.

TRAF2 $\mathrm{KO}$ in mice causes perinatal lethality that is prevented by deletion of TNFa or TNFR1. ${ }^{35,36}$ The lethal TRAF2 KO phenotype is diminished on a BALB/c background, though these mice succumb within 2 weeks after birth and display symptoms of inflammatory bowel disease. ${ }^{37}$ TRAF2deficient mice have exacerbated hepatocyte apoptosis and mortality upon injection with agonistic anti-Fas antibody. ${ }^{34}$ Given its involvement in modulating apoptotic cell death, we asked whether TRAF2 also plays a significant role in regulating necroptosis.

To approach this question, we used loss-of-function strategies, including siRNA knockdown and gene $\mathrm{KO}$, as well as some gain-of-function modalities. Mouse L929 fibroblasts displayed an augmented necroptotic response to TNFa, as did TRAF2 KO MEFs. Knockdown of RIPK1, RIPK3 or MLKL reversed this augmentation, confirming an effect of TRAF2 on necroptotic signaling. TRAF2 KO MEFs showed stronger necrosome formation than did WT MEFs, suggesting that at least one level of necroptosis suppression by TRAF2 is exerted upstream. One possible explanation for this enhanced necrosome formation is a diminished recruitment of clAPsand consequently-less RIPK1 ubiquitination, which would promote RIPK1 activation and necrosome assembly. ${ }^{2,23,28}$ However, phenotypic differences between TRAF2 versus cIAP1 KO MEFs suggested that TRAF2 may act also at some additional level(s). Several lines of evidence support this: first, endogenous TRAF2 and MLKL could be co-immunopreciptated in vitro and in vivo. Second, whereas TRAF2 and MLKL pre-associated in untreated cells, TNFa treatment induced their dissociation-in concert with increased recruitment of MLKL to RIPK3. Third, TRAF2 and MLKL bound to each other upon ectopic co-expression. Although the RING and CIM regions of TRAF2 were dispensable for MLKL binding, the C-terminal portion, which contains the TRAF homology motif, was important. This finding reinforces the conclusion that TRAF2 and MLKL interact in a biologically specific manner. Furthermore, immunofluorescence studies showed significant co-localization of TRAF2 and MLKL in nonstimulated cells and its reversal by TNFa. MLKL overexpression was sufficient to drive cell death as previously shown; ${ }^{14}$ consistent with the co-IP results, this was reversed by coexpression of WT TRAF2 and the RING and CIM but not the $\mathrm{C}$-terminal deletion variants. Together, these findings support the conclusion that TRAF2 exerts additional suppression of necroptosis at the level of MLKL. RIPK3 and MLKL showed significantly stronger baseline interaction in the absence of TRAF2, suggesting that TRAF2 and RIPK3 may compete for MLKL binding. Indeed, we observed substantially less binding of TRAF2 and MLKL upon co-transfection with RIPK3 in HEK293 cells (data not shown).

CYLD promotes necrosome formation by deubiquitinating RIPK1. ${ }^{29}$ Our data unravel an important additional 


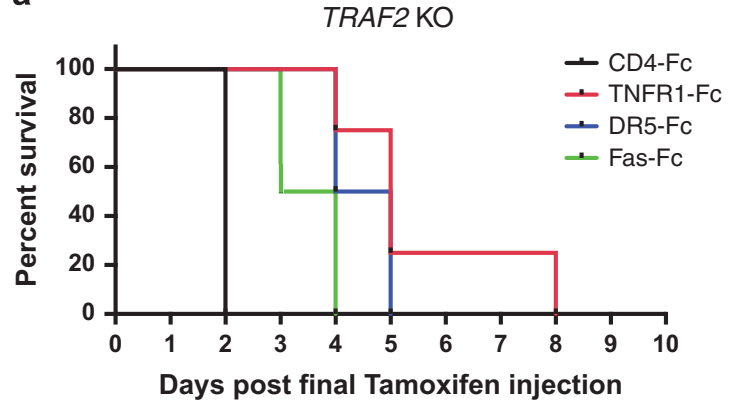

C

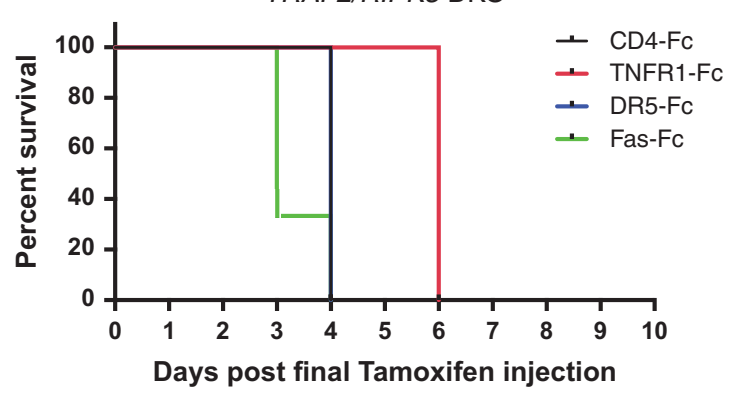

b

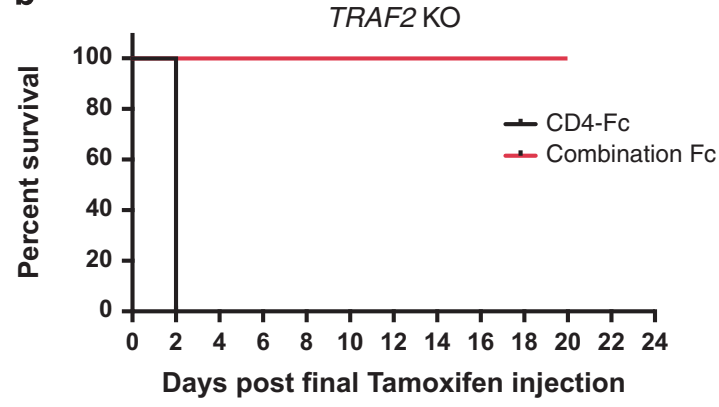

d

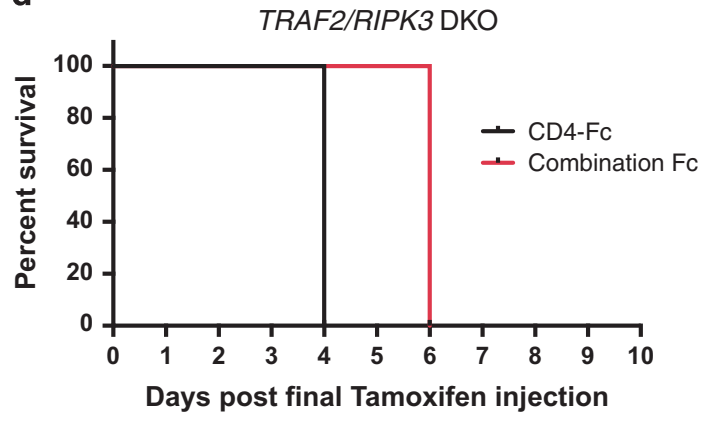

e

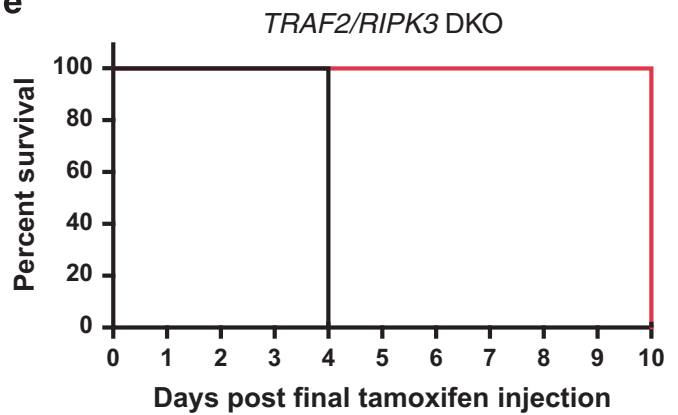

$-\mathrm{CD} 4-\mathrm{FC}$

- IFNAR1-Fc/TNFR1-Fc

f
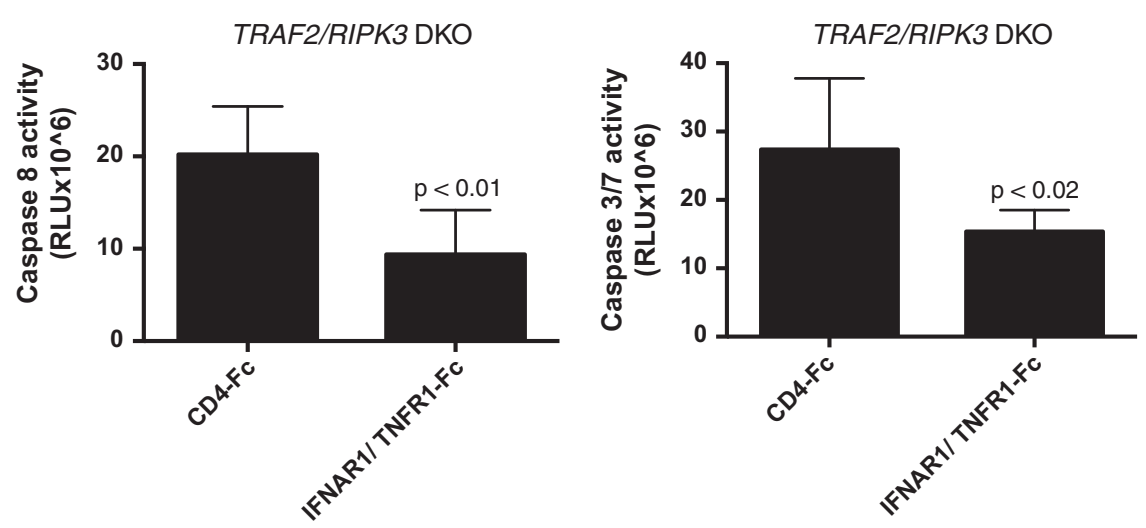

Figure 6 Death ligands differentially contribute to lethality in TRAF2 KO and TRAF2/RIPK3DKO mice. (a) TRAF2 KO mice were injected i.p. with tamoxifen (20 mg/kg) daily for 5 days. Mice were injected concurrently with $10 \mu \mathrm{g}$ per mouse each of CD4-Fc, TNFR1-Fc, DR5-Fc or Fas-Fc and observed for morbidity $(n=4, P=<0.0015)$. (b) TRAF2 $\mathrm{KO}$ mice were injected with tamoxifen as in a concurrently with control CD4-Fc (30 $\mu \mathrm{g}$ per mouse) or a combination of TNFR1-Fc, DR5-Fc and Fas-Fc (10 $\mu \mathrm{g}$ per mouse each) $(n=3,<0.05)$. (c) TRAF2/RIPK3DKO mice were treated as in a $(n=3, P=0.0113)$. (d) TRAF2/RIPK3 DKO mice were treated as in $\mathbf{b}(n=3, P=0.0253)$. (e, f) TRAF2/RIPK3 DKO mice were treated with tamoxifen as in a, concurrently with CD4-Fc (20 $\mu \mathrm{g}$ per mouse) or TNFR1-Fc and IFNAR1-Fc (10 $\mu$ g per mouse). Mice were followed for morbidity $(n=4, P=0.0082)(\mathbf{e})$. Intestines were collected on day 9 and extracts were analyzed by caspase-8 and caspase-3/7 activity assay (f). Bar graphs depict mean \pm S.E.M. $(n=4)$. $P$ values are by log-rank (Mantel-Cox) test (a-e) or Student's $t$ test (f) 
pro-necroptotic function of CYLD via dequbiquitination of TRAF2. In L929 cells, TRAF2 displayed baseline ubiquitination, which was reversed in a CYLD-dependent manner upon TNFa treatment. CYLD was also required for TNFa-induced dissociation of TRAF2 from MLKL and the consequent enhancement of necroptosis. In studies with purified recombinant proteins, clAP1-mediated ubiquitination of TRAF2 supported binding to MLKL, providing evidence for direct association, whereas CYLD-mediated deubiquitination of TRAF2 attenuated this interaction. Thus, CYLD-dependent TRAF2 deubiquitination reverses TRAF2-MLKL binding, perhaps permitting better MLKL engagement by the necrosome to trigger necroptosis.

Our in vivo findings support a role for TRAF2 in suppressing both apoptosis and necroptosis and demonstrate that this function is biologically critical. Whereas TRAF2 KO in adult mice led to rapid lethality, this was significantly delayed by $R I P K 3 \mathrm{KO}$, suggesting that unchecked necroptosis in the absence of TRAF2 contributes significantly to the observed mortality. Consistent with this, TRAF2 KO mice displayed spontaneous hepatic necrosome formation. It is likely that unchecked apoptosis also contributes to mortality upon TRAF2 KO, particularly in the absence of RIPK3. Supporting this, TRAF2/RIPK3 DKO mice showed a spontaneous increase in intestinal levels of caspase-8 protein and processing as well as enzymatic activity. A recent finding that may be related to this observation is that RIPK3 supports caspase-8 activation in a kinase-independent capacity. ${ }^{49}$ It is conceivable therefore that the observed death is primarily caused by excessive hepatic necroptosis in TRAF2 $\mathrm{KO}$ mice and by intestinal apoptosis in TRAF2/RIPK3 DKO mice. Although access to detailed histopathological analysis was not available to us in this study, it is notable that the DKO phenotype bears similarity to that reported recently for selective RIPK1 KO in intestinal epithelial cells. ${ }^{50-52}$ The latter involves kinaseindependent RIPK1 activity, which confers stabilization of several pro-survival proteins, including TRAF2, clAPs and cFLIP. ${ }^{52}$ It will be interesting to investigate whether TRAF2 is linked to the kinase-independent functions of RIPK1 and/or RIPK3.

Combined inhibition of the key death ligands TNFa, FasL and Apo2L/TRAIL using receptor-Fc decoys provided complete protection of TRAF2 KO but not TRAF2/RIPK3 DKO mice. This unexpected difference suggests that other pathological drivers may be involved in lethality upon deletion of both TRAF2 and RIPK3. Previous reports suggest that type I IFN upregulates caspase-8 transcription and promotes apoptosis independent of death ligands. ${ }^{45}$ Moreover, TRAF2 is implicated in signaling by IFNAR $1 .{ }^{53}$ In keeping with these findings, we observed diminished caspase-8 and caspase-3/7 activity in intestinal extracts from TRAF2 KO mice treated with TNFR1-Fc plus IFNAR1-Fc. Thus, in the absence of TRAF2, type I IFN signaling may be altered in a manner that augments apoptosis via caspase-8; other factors that remain to be identified further contribute to lethality in the added absence of RIPK3. In conclusion, our findings demonstrate that TRAF2 is an important biological suppressor of both apoptosis and necroptosis, affecting homeostasis of key organs and viability of the whole organism.

\section{Materials and Methods}

Cell culture. WT, TRAF2 KO, CIAP1 KO MEFs and L929 cells were cultured in Dulbecco's modified Eagle's medium. Culture media was supplemented with $10 \%$ fetal bovine serum and $2 \mathrm{mM} \mathrm{L-glutamine.}$

Experimental reagents. Abs for IP and IB analysis were TRAF2 (C-20, F-2), MLKL (Y-14), CYLD (E-10) and c-myc (9E10) from Santa Cruz, Dallas, TX, USA, RIPK1 (610459) from BD Bioscience (San Jose, CA, USA), RIPK3 (2283) from Pro-Sci (Poway, CA, USA), MLKL (clone 3H1) from Millipore (Billerica, MA, USA), ubiquitin (P4D1) from Cell Signaling (Danvers, MA, USA) and RIPK3 from Genentech (South San Francisco, CA, USA). TNF $\alpha$, and recombinant CD4-, TNFR1-, DR5-, Fas-, and IFNAR1-Fc fusion proteins ${ }^{54,55}$ were from Genentech. zVAD, cycloheximide, Necrostatin-1 and tamoxifen were from Sigma (St. Louis, MO, USA). Recombinant TRAF2 and MLKL were purchased from Novus (Littleton, CO, USA). Recombinant CIAP1 and CYLD were purchased from Boston Biochem (Cambridge, MA, USA).

Reagents, siRNA and cDNA transfection. The siRNA oligonucleotides against TRAF2, RIPK1, RIPK3, MLKL and CYLD were from Dharmacon (Lafayette, CO, USA).

Nontargeting siRNA control (siNTC, D-001810-01); GFP siRNA control (P-002048); TRAF2 (L-042814-01); RIP1 (L-040150-00); RIPK3 (L-049919-01); MLKL (L-061420-01); CYLD (L-055575-01). L929 cells were reverse-transfected using RNAiMAX from Thermo Fisher Scientific (Waltham, MA, USA). WT and TRAF2 KO MEFs were transfected using Genemute siRNA and plasmid transfection reagent from SignaGen Laboratories (Rockville, MD, USA), all according to the manufacturer's protocol.

The expression construct for the myc-tagged MLKL and FLAG-tagged WT TRAF2 and deletion mutants lacking the RING, CIM and TRAF domains were generated by DNA synthesis or site-directed mutagenesis. For ectopic expression experiments, L929 cells were transfected using Lipofectamine LTX Plus reagent (Thermo Fisher Scientific), while MEFs were transfected using Genemate transfection reagent, both according to the manufacturer's protocol.

IP and IB analysis. Cells were grown on $15 \mathrm{~cm}$ plates, treated as indicated, washed with PBS, and harvested with $1 \mathrm{ml}$ of the following lysis buffer: $20 \mathrm{mM}$ Tris $\mathrm{HCL}(\mathrm{pH} 7.5), 150 \mathrm{mM} \mathrm{NaCl}, 1.5 \%$ Triton $\mathrm{X}-100$, plus protease and phosphatase inhibitors from Roche Diagnostics (Indianapolis, IN, USA).

Cell extracts were left on ice for $20 \mathrm{~min}$ and centrifuged at 14000 r.p.m. for $15 \mathrm{~min}$. Five milligrams of protein lysate were used for IP using $2 \mu \mathrm{g}$ of the following antibodies: RIPK1 (610459), RIPK3 (Genentech), MLKL (clone 3H1) and TRAF2 (F-2) for overnight at $4^{\circ} \mathrm{C}$. The following day, $25 \mu$ l of protein $\mathrm{A} / \mathrm{G}$ ultralink resin from Thermo Fisher Scientific was added for $2 \mathrm{~h}$ at $4^{\circ} \mathrm{C}$, and the IPs were washed three times with lysis buffer, sample buffer was added, and beads were boiled for $5 \mathrm{~min}$ at $95^{\circ} \mathrm{C}$. Samples were then analyzed by SDS-PAGE followed by IB.

Analysis of ubiquitination. For analysis of total TRAF2 ubiquitylation, 1.5\% SDS and was added to cell lysates (5 mg protein), boiled for $15 \mathrm{~min}$ at $95^{\circ} \mathrm{C}$, diluted to $0.15 \%$ SDS lysis buffer and subjected to IP utilizing 10ug TRAF2 (F-2) IP antibody and $100 \mathrm{ul}$ protein A/G ultralink resin (Thermo Fisher Scientific) and then analyzed as above.

In vitro ubiquitination of TRAF2 and binding to MLKL. Recombinant TRAF2 ( $60 \mathrm{ng}$ ) was added to a ubiquitination reaction mix ( $30 \mu l$ ) containing E1, E2, CIAP1 and ubiquitin, as per manufacturer's instructions (Boston Biochem) and incubated for $1 \mathrm{~h}$. Recombinant MLKL $(60 \mathrm{ng})$ and CYLD $(60 \mathrm{ng})$ were added as indicated, and incubation was continued at $37^{\circ} \mathrm{C}$ for an additional $1 \mathrm{~h}$. To stop the reaction, $1 \mathrm{ml}$ lysis buffer containing TRAF2 antibody was added for $1 \mathrm{~h}$ at room temperature, followed by the addition of protein $A / G$ ultralink resin for another $1 \mathrm{~h}$. Beads were washed three times with lysis buffer, eluted and analyzed by SDS-PAGE.

Generation of inducible TRAF2/RIPK3 DKO mice. TRAF2 loxp/loxp RosaCre ERTS mice and RIPK3.neono.ko mice were generated as previously described ${ }^{56}$ To create TRAF2 KO/RIPK3 DKO mice, the two lines were crossed and mice were screened for TRAF2 loxp/loxp sites targeting exon 3 of TRAF2 and for Rosa-creERT2, as well as for RIPK3 KO.

To induce TRAF2 KO, tamoxifen was solubilized in sunflower oil and administered by intraperitoneal injection at the indicated dosages for five consecutive days followed 
by observation. Fc-fusion proteins were diluted in PBS at a concentration of $100 \mu \mathrm{g} / \mathrm{ml}$ and each mouse was dosed with $10 \mu \mathrm{g}$ total protein concurrently with tamoxifen by intraperitoneal injection.

All procedures were approved by and conformed to the guidelines and principles set by the Institutional Animal Care and Use Committee of Genentech and were carried out in an Association for the Assessmentand Accreditation of Laboratory Animal Care (AAALAC)-accredited facility.

Immunocytochemistry and confocal microscopy. L929 cells grown on Lab-Tekll chamberslides from Thermo Fisher Scientific were preincubated in the presence or absence of cycloheximide and $\mathrm{ZVAD}$ for $30 \mathrm{~min}$ at $37^{\circ} \mathrm{C}$ and then treated with or without TNF $\alpha$ for 90 min. Cultures were fixed with $4 \%$ paraformaldehyde for $15 \mathrm{~min}$ at room temperature and permeabilized with $0.2 \%$ saponin in blocking buffer (10\% goat serum, $10 \mathrm{mM}$ Hepes, $10 \mathrm{mM}$ glycine in RPMI 1640) for $15 \mathrm{~min}$ at room temperature. Slides were then washed and blocked in blocking buffer for $1 \mathrm{~h}$ at room temperature. Anti-MLKL $(1: 200)$, anti-TRAF2 $(1: 500)$ and anti-RIPK3 $(1: 50)$ were diluted in blocking buffer incubated with cells at $4{ }^{\circ} \mathrm{C}$ overnight. After three washes with PBS, cells were incubated with respective $1: 200$ diluted secondary antibodies conjugated with either Alexa488, Alexa555 or Alexa647 (Invitrogen). Slides were mounted with Prolong Gold anti-fade reagent containing DAPI from Thermo Fisher Scientific and viewed with a LEICA (Buffalo Grove, IL, USA) SP5 inverted confocal microscope under a $\times 63$ oil objective, using Leica LAS AF software to acquire images.

Quantitation of co-localized signal. Images were collected randomly (six images from each treatment; three independent experiments), and colocalization was determined with the 'Colocalization' Plug-in of ImageJ (NIH) with the same color-threshold settings for all six images from each treatment. The co-localized color channel (two of the three colors (red, green and blue)) were then counted with the 'Analyze particles' command of Image J. Percent colocalization was then calculated by total area of co-localized channel over total area of both channels.

Analysis of caspase-8 mRNA expression. Intestinal extracts were harvested and total RNA was extracted utilizing RNeasy Plus Mini kit (Qiagen, Valencia, CA, USA). RNA was converted to cDNA utilizing RT2 First Strand kit from Qiagen. Real-time PCR was then performed using RT2 QPCR primers for caspase8, actin and GAPDH (Qiagen) on an Applied Biosystems (Waltham, MA, USA) ViiA7 qPCR machine.

\section{Conflict of Interest}

The authors declare no conflict of interest.

Acknowledgements. We thank Eugene Varfolomeev and Domagoj Vucic for TRAF2 and CIAP1 KO MEFs, and Kim Newton and Vishva Dixit for TRAF2 and RIPK3 $\mathrm{KO}$ mice and for advice and comments. We also thank Genentech's mouse-facility staff for their support.

1. Zhang DW, Shao J, Lin J, Zhang N, Lu BJ, Lin SC et al. RIP3, an energy metabolism regulator that switches TNF-induced cell death from apoptosis to necrosis. Science 2009; 325: 332-336.

2. He S, Wang L, Miao L, Wang T, Du F, Zhao L et al. Receptor interacting protein kinase-3 determines cellular necrotic response to TNF-alpha. Cell 2009; 137: 1100-1111.

3. Ashkenazi A, Salvesen G. Regulated cell death: signaling and mechanisms. Annu Rev Cell Dev Biol 2014; 30: 337-356.

4. Salvesen GS, Ashkenazi A. Snapshot: caspases. Cell 2011; 147: 476-e1.

5. Cho YS, Challa S, Moquin D, Genga R, Ray TD, Guildford M et al. Phosphorylation-driven assembly of the RIP1-RIP3 complex regulates programmed necrosis and virus-induced inflammation. Cell 2009; 137: 1112-1123.

6. Zhao J, Jitkaew S, Cai Z, Choksi S, Li Q, Luo J et al. Mixed lineage kinase domain-like is a key receptor interacting protein 3 downstream component of TNF-induced necrosis. Proc Natl Acad Sci U S A 2012; 109: 5322-5327.

7. Robinson N, McComb S, Mulligan R, Dudani R, Krishnan L, Sad S. Type I interferon induces necroptosis in macrophages during infection with Salmonella enterica serovar Typhimurium. Nat Immunol 2012; 13: 954-962.

8. Mocarski ES, Upton JW, Kaiser WJ. Viral infection and the evolution of caspase 8-regulated apoptotic and necrotic death pathways. Nat Rev Immunol 2012; 12: 79-88.

9. Li J, McQuade T, Siemer AB, Napetschnig J, Moriwaki K, Hsiao YS et al. The RIP1/RIP3 necrosome forms a functional amyloid signaling complex required for programmed necrosis. Cell 2012; 150: 339-350.
10. Murphy JM, Czabotar PE, Hildebrand JM, Lucet IS, Zhang JG, Alvarez-Diaz S et al. The pseudokinase MLKL mediates necroptosis via a molecular switch mechanism. Immunity 2013; 39: 443-453.

11. Sun L, Wang H, Wang Z, He S, Chen S, Liao D et al. Mixed lineage kinase domain-like protein mediates necrosis signaling downstream of RIP3 kinase. Cell 2012; 148: 213-227.

12. Wang H, Sun L, Su L, Rizo J, Liu L, Wang LF et al. Mixed lineage kinase domain-like protein MLKL causes necrotic membrane disruption upon phosphorylation by RIP3. Mol Cell 2014; 54: $133-146$.

13. Galluzzi L, Kepp $O$, Kroemer G. MLKL regulates necrotic plasma membrane permeabilization. Cell Res 2014; 24: 139-140.

14. Dondelinger Y, Declercq W, Montessuit S, Roelandt R, Goncalves A, Bruggeman I et al. MLKL compromises plasma membrane integrity by binding to phosphatidylinositol phosphates. Cell Rep 2014; 7: 971-981.

15. Chen X, Li W, Ren J, Huang D, He WT, Song Y et al. Translocation of mixed lineage kinase domain-like protein to plasma membrane leads to necrotic cell death. Cell Res 2014; 24: 105-121.

16. Zhou W, Yuan J. Necroptosis in health and diseases. Semin Cell Dev Biol 2014; 35: 14-23.

17. Linkermann A, Hackl MJ, Kunzendorf U, Walczak H, Krautwald S, Jevnikar AM. Necroptosis in immunity and ischemia-reperfusion injury. Am J Transplant 2013; 13: 2797-2804.

18. Linkermann A, Brasen JH, Himmerkus N, Liu S, Huber TB, Kunzendorf $\mathrm{U}$ et al. Rip1 (receptor-interacting protein kinase 1) mediates necroptosis and contributes to renal ischemia/reperfusion injury. Kidney Int 2012; 81: 751-761.

19. Welz PS, Wullaert A, Vlantis K, Kondylis V, Fernandez-Majada V, Ermolaeva M et al. FADD prevents RIP3-mediated epithelial cell necrosis and chronic intestinal inflammation. Nature 2011; 477: 330-334.

20. Oberst A, Dillon CP, Weinlich R, McCormick LL, Fitzgerald P, Pop C et al. Catalytic activity of the caspase-8-FLIP(L) complex inhibits RIPK3-dependent necrosis. Nature 2011; 471: 363-367.

21. Kaiser WJ, Upton JW, Long AB, Livingston-Rosanoff D, Daley-Bauer LP, Hakem R et al. RIP3 mediates the embryonic lethality of caspase-8-deficient mice. Nature 2011; 471: 368-372.

22. Bonnet MC, Preukschat D, Welz PS, van Loo G, Ermolaeva MA, Bloch W et al. The adaptor protein FADD protects epidermal keratinocytes from necroptosis in vivo and prevents skin inflammation. Immunity 2011; 35: 572-582.

23. Vanlangenakker N, Vanden Berghe $\mathrm{T}$, Bogaert $\mathrm{P}$, Laukens $\mathrm{B}$, Zobel K, Deshayes $\mathrm{K}$ et al. CIAP1 and TAK1 protect cells from TNF-induced necrosis by preventing RIP1/RIP3dependent reactive oxygen species production. Cell Death Differ 2011; 18: 656-665.

24. O'Donnell MA, Perez-Jimenez E, Oberst A, Ng A, Massoumi R, Xavier R et al. Caspase 8 inhibits programmed necrosis by processing CYLD. Nat Cell Biol 2011; 13: 1437-1442.

25. Wu J, Huang Z, Ren J, Zhang Z, He P, Li Y et al. Mlkl knockout mice demonstrate the indispensable role of Mlkl in necroptosis. Cell Res 2013; 23: 994-1006.

26. Bertrand MJ, Milutinovic S, Dickson KM, Ho WC, Boudreault A, Durkin J et al. clAP1 and CIAP2 facilitate cancer cell survival by functioning as E3 ligases that promote RIP1 ubiquitination. Mol Cell 2008; 30: 689-700.

27. Rodrigue-Gervais IG, Labbe K, Dagenais M, Dupaul-Chicoine J, Champagne C, Morizot A et al. Cellular inhibitor of apoptosis protein clAP2 protects against pulmonary tissue necrosis during influenza virus infection to promote host survival. Cell Host Microbe 2014; 15: 23-35.

28. Jacquel A, Auberger P. cIAPs and XIAP reduce RIPKs to silence. Blood 2014; 123 2445-2446.

29. Moquin DM, McQuade T, Chan FK. CYLD deubiquitinates RIP1 in the TNFalpha-induced necrosome to facilitate kinase activation and programmed necrosis. PLoS One 2013; 8: e76841.

30. Vince JE, Pantaki D, Feltham R, Mace PD, Cordier SM, Schmukle AC et al. TRAF2 must bind to cellular inhibitors of apoptosis for tumor necrosis factor (tnf) to efficiently activate nf-\{kappa\}b and to prevent tnf-induced apoptosis. J Biol Chem 2009; 284: 35906-35915.

31. Shu HB, Takeuchi M, Goeddel DV. The tumor necrosis factor receptor 2 signal transducers TRAF2 and c-IAP1 are components of the tumor necrosis factor receptor 1 signaling complex. Proc Natl Acad Sci U S A 1996; 93: 13973-13978.

32. Hsu H, Shu HB, Pan MG, Goeddel DV. TRADD-TRAF2 and TRADD-FADD interactions define two distinct TNF receptor 1 signal transduction pathways. Cell 1996; 84: 299-308.

33. Rothe M, Sarma V, Dixit VM, Goeddel DV. TRAF2-mediated activation of NF-kappa B by TNF receptor 2 and CD40. Science 1995; 269: 1424-1427.

34. Gonzalvez F, Lawrence D, Yang B, Yee S, Pitti R, Marsters S et al. TRAF2 Sets a threshold for extrinsic apoptosis by tagging caspase-8 with a ubiquitin shutoff timer. Mol Cell 2012; 48: 888-899.

35. Yeh WC, Shahinian A, Speiser D, Kraunus J, Billia F, Wakeham A et al. Early lethality, functional NF-kappaB activation, and increased sensitivity to TNF-induced cell death in TRAF2-deficient mice. Immunity 1997; 7: 715-725.

36. Lee SY, Reichlin A, Santana A, Sokol KA, Nussenzweig MC, Choi Y. TRAF2 is essential for JNK but not NF-kappaB activation and regulates lymphocyte proliferation and survival. Immunity 1997; 7: 703-713.

37. Piao JH, Hasegawa M, Heissig B, Hattori K, Takeda K, Iwakura Y et al. Tumor necrosis factor receptor-associated factor (TRAF) 2 controls homeostasis of the colon to prevent 
spontaneous development of murine inflammatory bowel disease. J Biol Chem 2011; 286: 17879-17888.

38. Karl I, Jossberger-Werner M, Schmidt N, Horn S, Goebeler M, Leverkus $M$ et al. TRAF2 inhibits TRAIL- and CD95L-induced apoptosis and necroptosis. Cell Death Dis 2014; 5: e1444.

39. Vandenabeele P, Galluzzi L, Vanden Berghe T, Kroemer G. Molecular mechanisms of necroptosis: an ordered cellular explosion. Nat Rev Mol Cell Biol 2010; 11: 700-714.

40. Gentle IE, Wong WW, Evans JM, Bankovacki A, Cook WD, Khan NR et al. In TNF-stimulated cells, RIPK1 promotes cell survival by stabilizing TRAF2 and cIAP1, which limits induction of non-canonical NF-kappaB and activation of caspase-8. J Biol Chem 2011; 286: 13282-13291.

41. Takeuchi M, Rothe M, Goeddel DV. Anatomy of TRAF2. Distinct domains for nuclear factorkappaB activation and association with tumor necrosis factor signaling proteins. J Biol Chem 1996; 271: 19935-19942.

42. Sondarva G, Kundu CN, Mehrotra S, Mishra R, Rangasamy V, Sathyanarayana P et al. TRAF2-MLK3 interaction is essential for TNF-alpha-induced MLK3 activation. Cell Res 2010; 20: 89-98.

43. Kovalenko A, Chable-Bessia C, Cantarella G, Israel A, Wallach D, Courtois G. The tumour suppressor CYLD negatively regulates NF-kappaB signalling by deubiquitination. Nature 2003; 424: 801-805.

44. Trompouki E, Hatzivassiliou E, Tsichritzis T, Farmer H, Ashworth A, Mosialos G. CYLD is a deubiquitinating enzyme that negatively regulates NF-kappaB activation by TNFR family members. Nature 2003; 424: 793-796.

45. Lietke CG N, Manns M, Trautwein C. Interferon-alpha enhances TRAIL-mediated apoptosis by up-regulating caspase-8 transcription in human hepatoma cells. J Hepatol 2006; 44 342-349.

46. Linkermann A, Green DR. Necroptosis. N Engl J Med 2014; 370: 455-465.
47. Hsu H, Huang J, Shu HB, Baichwal V, Goeddel DV. TNF-dependent recruitment of the protein kinase RIP to the TNF receptor-1 signaling complex. Immunity 1996; 4: 387-396.

48. Wong WW, Gentle IE, Nachbur U, Anderton H, Vaux DL, Silke J. RIPK1 is not essential for TNFR1-induced activation of NF-kappaB. Cell Death Differ 2010; 17: 482-487.

49. Newton K, Dugger DL, Wickliffe KE, Kapoor N, de Almagro MC, Vucic D et al. Activity of protein kinase RIPK3 determines whether cells die by necroptosis or apoptosis. Science 2014; 343: 1357-1360.

50. Rickard JA, O'Donnell JA, Evans JM, Lalaoui N, Poh AR, Rogers T et al. RIPK1 regulates RIPK3-MLKL-driven systemic inflammation and emergency hematopoiesis. Cell 2014; 157: $1175-1188$.

51. Takahashi N, Vereecke L, Bertrand MJ, Duprez L, Berger SB, Divert T et al. RIPK1 ensures intestinal homeostasis by protecting the epithelium against apoptosis. Nature 2014; 513: 95-99.

52. Dannappel M, Vlantis K, Kumari S, Polykratis A, Kim C, Wachsmuth L et al. RIPK1 maintains epithelial homeostasis by inhibiting apoptosis and necroptosis. Nature 2014; 513: 90-94.

53. Yang $\mathrm{CH}$, Murti A, Pfeffer SR, Fan M, Du Z, Pfeffer LM. The role of TRAF2 binding to the type I interferon receptor in alternative NF kappaB activation and antiviral response. J Biol Chem 2008; 283: 14309-14316.

54. Ashkenazi A, Marsters SA, Capon DJ, Chamow SM, Figari IS, Pennica D et al. Protection against endotoxic shock by a tumor necrosis factor receptor immunoadhesin. Proc Natl Acad Sci U S A 1991; 88: 10535-10539.

55. Ashkenazi A, Chamow SM. Immunoadhesins as research tools and therapeutic agents. Curr Opin Immunol 1997; 9: 195-200.

56. Newton K, Sun X, Dixit VM. Kinase RIP3 is dispensable for normal NF-kappa Bs, signaling by the B-cell and T-cell receptors, tumor necrosis factor receptor 1 , and Toll-like receptors 2 and 4. Mol Cell Biol 2004; 24: 1464-1469.

Supplementary Information accompanies this paper on Cell Death and Differentiation website (http://www.nature.com/cdd) 